\title{
Cognição situada e a cultura da aprendizagem: algumas considerações
}

\section{Cristina Azra Barrenechea*}

\begin{abstract}
RESUMO
Este trabalho comenta as declarações levantadas no artigo de Brown, Collins e Duguid's, intitulado "Situated Cognition and the Culture of Learning" (1989). Nesse artigo, os autores apresentam um modelo para ensinar o conhecimento dentro de contexto de funcionamento. Eles também sugerem que os métodos usados na educação não são efetivos para uma aprendizagem robusta e duradoura. O presente trabalho discute algumas das evidencias desse estudo apontando para a necessidade de um estudo mais extensivo não apenas nos modelos de aplicação como também em sua fundamentação teórica. De acordo com os autores, a cognição do conhecimento tem uma natureza situada porque partes importantes dela são implícitas ao seu contexto de atividade. A educação atual esquematiza conceitos gerais de um conhecimento para facilitar sua explicação para os alunos. No entanto, essa forma de ensinar um conhecimento é muito abstraída da situação real que produziu este conhecimento. Por isso, os alunos terminam aprendendo um tipo passivo de conhecimento, pois eles não aprendem como ele funciona em uma situação real. Assim, pela falta de conhecer o contexto no qual um conhecimento opera, os alunos não aprendem a aplicar aquele conhecimento propriamente. O contexto de atividade de um conhecimento detém importantes aspectos de conhecimento implícitos em uma forma cultural de ver, produzir e usar as ferramentas. Ao experienciar todos estes aspectos, os alunos podem construir modelos operativos para produzir um tipo de conhecimento ativo, que é abertamente influenciado pelo contexto de aplicação. Os autores sustentam que o conhecimento deveria ser ensinado em uma visão integrada de seu contexto, atividade, ferramentas e cultura, que têm um importante papel na cognição deste conhecimento.
\end{abstract}

Palavras-chave: ciência cognitiva, aprendizagem, educação ancorada.

* Professora do Setor de Educação, da UFPR. cristinab2001@yahoo.com.br 


\begin{abstract}
This work comments on the claims raised on Brown, Collins and Duguid's article "Situated Cognition and the Culture of Learning" ( 1989 ). In that article, the authors present a model for approaching knowledge on a more work like context. They also suggest that the way schooling methods approach knowledge are not effective for an enduring learning. This work discusses some of the main evidences of their study pointing at the need of a more thorough study not only on workable models but also on theoretical framework. According to the authors, the cognition of a knowledge has a situated nature because its important parts are placed in its context of activity. Current education schematize general concepts of a knowledge to make its explanation easier to the students. However, this way of teaching a knowledge is too abstracted from the real situation that produced this knowledge. Therefore students end up learning a passive kind of knowledge for they don't learn how it works in a real situation. Hence, by lacking to know the context in which knowledge works, students don't learn how to apply that knowledge properly. The context of knowledge activity holds important features of the know-ledge that are implicit in the cultural perceptions of practitioners, which produce and use the tools. By experiencing all of these aspects, students can build operating models to produce an active kind of knowledge, that is openly influenced by its application's context. The authors claim that knowledge should be taught in an integrated view of its context, activity, tools and culture, which have an important role on the knowledge's cognition.

Key-words: cognitive science, learning, anchored instruction.
\end{abstract}

\title{
Introdução
}

Brown, Collins e Duguid são precursores do modelo teórico da Aprendizagem Ancorada. Em seu artigo "Situated Cognition and the Culture of Learning" (1989), eles discutem os pressupostos desta teoria, sustentando que a cognição do conhecimento tem uma natureza situada porque partes importantes dela são implícitas ao seu contexto de atividade. Segundo os autores, a educação atual esquematiza conceitos gerais de um conhecimento para facilitar sua explicação para os alunos. No entanto, essa forma de ensinar um conhecimento é muito abstraída da situação real que produziu este conhecimento. Por isso, os alunos terminam aprendendo um tipo passivo de conhecimento, pois eles não aprendem na forma como ele funciona em uma si- 
tuação real. Pela falta de conhecer o contexto no qual um conhecimento opera, os alunos não aprendem a aplicar aquele conhecimento propriamente. $\mathrm{O}$ contexto de atividade de um conhecimento detém importantes aspectos de conhecimento implícitos em uma forma cultural de ver, produzir e usar as ferramentas. Ao experienciar todos estes aspectos, os alunos podem construir modelos operativos para produzir um tipo de conhecimento ativo, que é abertamente influenciado pelo contexto de aplicação. Os autores sustentam que o conhecimento deveria ser ensinado em uma visão integrada de seu contexto, atividade, ferramentas e cultura, que têm um importante papel na cognição deste conhecimento.

Nesse artigo, os autores examinam o que sustenta um aprendizado duradouro e oferecem novas soluções para gerar métodos mais efetivos de ensino. Para tanto, eles analisam algumas características fundamentais do conhecimento e seu papel na aprendizagem de um dado domínio de conhecimento. Características tais como a cultura de um conhecimento, suas ferramentas, atividades e contexto social são declaradas como chaves para a mudança dos métodos de ensino formal como nós os conhecemos hoje. Assim, os autores analisam os relacionamentos entre estes aspectos do conhecimento e posteriormente apresentam algumas evidências do seu impacto nos métodos de ensino eficazes. Eles ilustram algumas características dos processos cognitivos da aprendizagem em dois exemplos do ensino de matemática: o ensino de Schoenfeld, de resolução de problemas, e o ensino de Lampert, de multiplicação. Finalmente, os autores sugerem a adoção de um novo paradigma para as práticas no ensino formal chamado "aprendizagem cognitiva". Esse processo de ensino é baseado em atividades que são tão autenticas quanto aquelas feitas na cultura de domínio do conhecimento. Essa abordagem enfatiza práticas de modelagem, trabalho colaborativo, enculturação e usa a heurística nas estratégias de resolução de problemas.

\section{Algumas considerações}

Em métodos tradicionais de ensino, o conhecimento tem sido separado do contexto no qual ele é produzido. A situação e atividade de um conhecimento produz a cognição de conceitos funcionais deste conhecimento. $\mathrm{O}$ conhecimento ensinado pela educação atual não oferece atividades e ferramentas relacionadas à cultura de campo de um dado conhecimento. $\mathrm{O}$ uso de 
abstrações conceituais no ensino formal não oferece um aprendizado duradouro porque elas não são discutidas dentro do contexto no qual o aprendizado será aplicado. Este processo leva a uma lacuna nos resultados do aprendizado, que se situa na diferença entre saber a respeito de algo e saber fazer algo. Ao examinar o relacionamento entre o aprendizado, contexto do conhecimento, atividades, ferramentas e sua cultura, os autores sugerem uma nova abordagem de ensino chamada Aprendizagem ${ }^{1}$ Cognitiva.

\section{Conhecimento situado e aprendizagem}

A cognição de um conhecimento tem uma natureza situada porque existem partes relevantes para seu entendimento que se encontram no contexto de atividade deste conhecimento. Estas partes no entanto são subjacentes à cultura do conhecimento e ao sistema de valores que esta cultura emprega para usar o conhecimento em diferentes situações. A educação atual esquematiza os elementos gerais de um conhecimento a fim de facilitar sua explicação. No entanto, a abstração de conceitos para ensinar um dado conhecimento dissocia aspectos importantes que estão relacionados à sua aplicação em condições variáveis e diversificadas. Assim, o conhecimento de um conteúdo é ensinado separadamente do conhecimento de como aplicar este conteúdo e de como ele funciona. Métodos atuais de ensino, por isso, falham em permitir aos alunos experienciar e construir modelos operacionais e conceitos indexadores para aplicar tais modelos. Os autores fundamentam esta declaração com um estudo sobre o aprendizado do significado das palavras e seus usos contextuados. O estudo de MILLER e GILDEA (1987) evidenciou como o aprendizado das palavras é dependente de um contexto de uso, o qual é indexador do significado da palavra, ao invés da conceitualização do dicionário. De acordo com o comentário de PALINCSAR (1986), estudantes iniciantes podem ser, à princípio, ineptos para usar dicionários apropriadamente, no entanto, a longo prazo, eles podem utilizá-los como uma "ferramenta para abordar uma ampla variedade de atividades autênticas.” (PALINCSAR, 1986). Ela nos lembra que o que faz o uso habilidoso de um dicionário e a aplicação apropriada das palavras em diferentes contextos e situações é uma "bagagem extensiva de conhecimento", a qual, de qualquer modo, deve vir de uma experiência extensiva de cognição situada na aplicação das palavras.

1 A tradução de aprendizagem vem no contexto de Apprenticeship, é um termo usado para designar o processo de aprendizado ou treinamento dos artesãos e dos aprendizes em um ofício. 
WINERBURG declara que este artigo está orientado para descrever os métodos de ensino formal como tudo o que há de ruim enquanto que este método de aprendizagem oferece tudo de bom. Ele chama atenção para os aspectos da ambigüidade, contradições e incoerências em ambos os lados que não estão claramente discutidos no artigo. Ele também apoia sua observação no trabalho de BERKHOFER (1978), que descreve a atitude romântica ao criticar as instituições prevalecentes.

\section{Aprendizagem e ferramentas}

O uso de ferramentas dentro de uma cultura de conhecimento determina como os praticantes vêem o mundo. O uso apropriado de ferramentas está em função da cultura e das atividades nas quais as ferramentas são desenvolvidas. A cultura, dessa forma, determina como a ferramenta será usada. Para usar a ferramenta propriamente, alunos necessitam conhecer a cultura à qual a ferramenta pertence. Eles necessitam aprender a cultura das pessoas que usam tais ferramentas de forma que eles possam aprendê-las apropriadamente, porque aprender a usar estes instrumentos é um processo contínuo em utilizá-los em muitas situações. No entanto, na educação corrente, é esperado dos alunos o uso de ferramentas de um domínio sem que eles possam adotar sua cultura. Conceitos de um conhecimento também são ferramentas, porque eles são produzidos diretamente do contexto de atividades de uma cultura. Conhecimento conceitual, então, não pode ser abstraído de uma cultura e suas atividades, porque conceitos são de fato um conjunto de ferramentas que estão situados e são progressivamente desenvolvidos através de atividade. Cultura, ferramentas, conceitos e atividades são interdependentes como corpo de conhecimento.

Tanto PALINCSAR quanto WINERBURG criticam o caso do observador do peso, usado como um exemplo para ilustrar como o contexto de atividade pode ajudar a solucionar um problema de matemática. Eles mostram que a solução executada pelo observador de peso não utilizou nenhuma habilidade matemática ou conhecimento prévio para resolver o problema, ao contrário, evitou-as com o uso do ambiente em si. Eles não acharam este caso muito apropriado para apoiar a evidência de que esta pessoa estava tirando qualquer aprendizado autêntico desta prática. Sugerimos que os autores deveriam utilizar este exemplo para demonstrar como alunos que aprendem pela educação formal, ou da forma escolar, estão despreparados para aplicar seu conhecimento matemático em práticas no mundo real. A questão central tirada deste comentário diz respeito à transferência de conhecimento para além da situação na qual o conhecimento foi aprendido. A questão colocada por WINERBURG é chave para o valor da cognição situada para transferências posteriores: o ob- 
servador de peso seria capaz de generalizar o tipo de solução criativa para outras situações? Em nossa perspectiva, qualquer aprendizado que tenha ocorrido em uma situação de atividade é robusto o bastante para ser transferido para além daquela situação, caso o aprendiz seja capaz de generalizar este aprendizado dentro de um modelo conceitual. A questão de PALINCSAR e WINERBURG levanta um ponto muito interessante para os objetivos do aprendizado situado e o seu papel em um processo de longo termo.

WINERBURG também aponta para a importância do ensino de conceitos abstratos para tornar as crianças capazes de generalizarem noções que elas aprendem. Ele fundamenta sua visão com três estudos: SANDIESON (1988), CASE;, GRIFFIN e LEHMAN, LEMPERT e NISBETT (1988). Nosso entendimento sobre esta questão é de que conceitos abstratos e conhecimento situado não necessariamente se excluem entre si, ao contrário, eles são complementares no processo de aprendizagem. Esta questão pede por estudos que determinem em que proporção, e em que momento, no processo de aprendizagem, as abstrações conceptuais sobre um dado conhecimento se fazem necessárias, e como elas afetam a habilidade de transferencia e generalização da aprendizagem pelo aluno. Sendo a aprendizagem uma experiência tão vasta e rica, acreditamos que ela permite espaço para muitas abordagens de acordo com as diferentes demandas do processo cognitivo. O uso isolado de uma ou outra abordagem (abstrata ou situada) é ensino pobre.

\section{Aprendizagem e enculturação}

A escola leva os alunos a se engajarem em culturas acadêmicas de atividade que diferem da cultura relevante do domínio do conhecimento. No entanto, tais culturas escolares não podem ensinar como usar as ferramentas conceituais como elas são usadas na prática autêntica. Alunos necessitam ser expostos ao uso de ferramentas conceituais da forma como elas são utilizadas na prática autêntica. Ao fazê-lo, eles adotariam o comportamento e sistema de valores de novos grupos sociais e aprenderiam elementos da cultura ambiente, ao invés do ensino explicitado. $\mathrm{O}$ aprendizado do uso de conceitos e de ferramentas implica numa mudança na visão de mundo do aprendiz, através de um sistema de crenças culturais no qual as ferramentas são utilizadas. Não estariam os autores superestimando o papel da enculturação no processo de aprendizagem, a menos que se demonstre formas exeqüíveis de aplicação destes pressupostos nas abordagens curriculares? 


\section{A atividade autêntica}

É vista como um processo de enculturação porque ela pertence à cultura de cada domínio do conhecimento. Os autores desenvolvem esta definição para demarcar a diferença entre a atividade autêntica de um campo de domínio e a atividade que é praticada dentro da cultura da escola, a qual acultura aspectos importantes da interação social e do entendimento do uso situado das ferramentas.

WINERBURG demonstrou que os autores apenas apresentaram experiências bem sucedidas do ensino de matemática como exemplos para ilustrar sua hipótese. No entanto, enculturar em matemática é mais fácil do que enculturar em história porque o sistema de crenças da história e as suas práticas profissionais não têm uma natureza de "resolução de problemas" como a matemática. Além disso, ainda que o artigo mostre como as práticas da escola estão longe da cultura de um dado conhecimento, este fato em si não é suficiente para sugerir que o uso na escola das mesmas práticas que os especialistas usam em seu lugar de trabalho tornará o ensino mais efetivo.

PALINCSAR também concorda que eles superestimam o papel da enculturação de um sistema de crenças como uma condição essencial para o aprendizado efetivo de ferramentas conceituais. Em ambas as críticas encontramos o questionamento: a encultaração é tão essencial para a cognição situada ocorrer? O que esperar do sistema de crenças em um processo de enculturação?

Ao examinar estas críticas, acreditamos que o processo de enculturação é a questão mais problemática do artigo. Enquanto os objetivos da enculturação e seu modelo conceitual não forem definidos, eles variarão com as demandas, objetivos e natureza epistemológica de cada disciplina curricular. PALINCSAR, por exemplo, orienta a questão para o que, exatamente, na cultura de um praticante, é relevante para os alunos enculturarem. Ela também questiona a necessidade real da enculturação para que os alunos aprendam a usar uma ferramenta. De fato, os autores não parecem ter fundamentado a necessidade de enculturação em situações mais específicas da educação para demonstrar porque e como isto poderia ser feito. PALINCSAR conclui sugerindo alguns objetivos da enculturação, tais como autopercepção, conhecimento prévio, controle do comportamento, interação social e entendimento de como os alunos deveriam aprender antes que eles possam esperar usar as ferramentas com a expertise de praticantes experimentados. Ela questiona como adotar a aprendizagem situada como um método bem sucedido e operante dentro do currículo. 


\section{Evidência}

\section{Atividade de estudantes, praticantes e pessoas comuns}

Baseado nos estudos etnográficos de Jean LAVE (1988b), o autor mostra que as escolas têm sua própria cultura e isto é o ponto de partida para o entendimento dos problemas de aprendizagem na escola. A cultura da escola usa práticas de aprendizagem que não oferecem significado e proposta para o que é aprendido. O estudo de LAVE examina quão distinta dos métodos de escolarização é a forma como pessoas comuns usam para aprender. Seu estudo compara três tipos de aprendizes: pessoas comuns, estudantes e praticantes. As pessoas comuns aprendem um conjunto de práticas ao enculturarem o processo do aprendiz de ofício em uma prática comunitária. Aos estudantes, de outro modo, é requerida uma mudança de comportamento quando eles entram dentro de um ambiente de escolarização. Nele, o aprendizado é feito através de problemas bem definidos, definições formais e atividades de manipulação de símbolos ao invés do uso de estratégias gerais para uma dedução intuitiva na resolução de questões e a negociação de significados nas atividades do dia-a-dia.

Pessoas comuns e praticantes executam suas atividades dentro de culturas nas quais eles trabalham e desenvolvem seu processo de aprendizagem. Essas atividades dão os parâmetros para os problemas e questões com as quais eles lidam. A cognição envolvida em um problema tanto quanto em sua solução não deveria ser vista separada do contexto que a produziu, o qual pode funcionar como um andaime para a resolução dos problemas.

PALINCSAR coloca que o estudo de como as pessoas comuns aprendem não é uma boa evidência para fundamentar o valor da aprendizagem do aprendiz de ofícios como um método de aprendizagem mais efetivo, porque as pessoas comuns e aprendizes não sabem e não entendem o uso das ferramentas e nem como abordar problemas da forma como os praticantes o fazem. Portanto, eles não possuem práticas similares. Ela pede evidências mais efetivas e questiona a hipótese de que a cultura da escola torna o aprendizado frágil: são pessoas comuns e praticantes iguais? Qual é a diferença entre eles?

A cognição sempre pede por um contexto para escorar seus processos e buscar soluções. Dentro da cultura escolar, as situações nas quais o conhecimento é praticado são muito aperfeiçoadas, bem definidas e especializadas. Ainda que os alunos se apoiem em dicas e estruturas que ocorrem na situação dos livros-texto, este tipo de estratégia não os ajudará posteriormente em situações no mundo real, onde os problemas não são apresentados como eles o 
são nos livros-texto. De acordo com a crítica de WINERBURG esta questão tem sido vista desde 1893, quando a Associação Nacional de Educação formou o Comitê dos Dez. Indiretamente ele aponta para a falta de referências na literatura relacionada. Adicionalmente, os estudantes deveriam ser motivados para usarem sua própria heurística para abordar a resolução de problemas.

\section{Estruturando a atividade}

A atividade autêntica permite ao aprendiz o acesso ao mesmo tipo de entendimento que os praticantes têm. Os autores não explicaram porque a atividade impacta os usos das ferramentas e como isso acontece. A atividade fornece experiência, que é indexadora para ações subseqüentes. Representações produzidas por ação não podem ser substituídas por instrução declarativa sozinha. A cognição que resulta de ações torna-se um referencial central tanto na aprendizagem como na própria atividade. O uso de ferramentas pode determinar como uma pessoa percebe a atividade e, portanto, como uma pessoa aprende e age. Cada atividade cria representações indexadoras que, como a linguagem, apenas têm significado no contexto. Então, como a transferência para além do contexto ocorre? Como a generalização é feita? Como este aprendizado pode ser cheio de significado para ajudar outras situações? Os autores não deixam claro como este tipo de aprendizagem é processado e reutilizado em situações posteriores. O conhecimento indexa suas atividades e ambiente, que oferecem partes essenciais de sua estrutura e sentido, alguns dos quais estão na mente, e outros, no mundo. "A estrutura da cognição está amplamente distribuída através do ambiente, e baseia as representações indexadoras que as pessoas formam na atividade" (p. 14) . Estas representações indexadoras ajudam as tarefas subseqüentes, as quais não podem ser facilmente declaradas (descritas ou relembradas na ausência da situação). De novo, a noção de representações indexadoras que as pessoas formam de sua atividade situada não está clara, ela necessita a descrição de como estas representações acontecem e como elas funcionam no processo de aprendizagem de uma cognição situada.

\section{Aprendizado através de Aprendizagem (Apprentiscehip) ${ }^{2}$ Cognitiva}

Métodos prevalecentes de escolarização partem do pressuposto que o conhecimento é individual e auto-estruturado, que os conceitos são abstratos,

2 Apprenticeship é um termo usado para designar o processo de aprendizado dos artesãos, o processo de iniciação dos aprendizes em um ofício. 
relativamente fixos e não afetados pela atividade que os produziu, que a cultura da escola realmente influencia o que é aprendido e que o comportamento de pessoas comuns deveria ser desencorajado. Os autores propõem o uso de métodos de aprendizagem cognitiva para enculturarem os alunos dentro de práticas autênticas similares às atividades e interações sociais do aprendizado de um ofício. Eles tentam fundamentar esta afirmação ao ilustrarem com algumas das características do processo da aprendizagem cognitiva dos aprendizes de ofício em dois exemplos do ensino de matemática

a) O ensino de Schoenfeld de resolução de problemas, o qual é um processo de ensino baseado em trabalho colaborativo envolvendo a solução de problemas e examinando a cultura e práticas do pensamento matemático. Procedimentos para a resolução de problemas tais como o trabalho a partir de uma solução inicial, o uso sistemático de geração de procedimentos, tendo mais do que uma forma de solucionar um problema, são ilustrados em uma ação ao invés de declarados pelo professor. Estas práticas mostram para os alunos como os matemáticos olham para os problemas (como numa busca para construir significações) na prática profissional.

b) A multiplicação do ensino de Lampert - Este método leva a que o entendimento implícito que os alunos têm do mundo seja utilizado em sala de aula, na construção de atividades e cultura para alcançar o aprendizado de algoritmos. Alunos trabalham através de quatro diferentes tipos de conhecimento matemático: a) Conhecimento intuitivo, o tipo de atalho espontâneo para solucionar problema em situações autênticas; b) Conhecimento computacional, a abordagem comum para ensinar algoritmos; c) Conhecimento concreto, uso de modelos concretos de algoritmos incrustados em histórias criadas por alunos; d) Conhecimento de princípios, princípios usados nas práticas dos algoritmos: associatividade e comutatividade. Ao usar e entender a conexão entre estes quatro tipos de conhecimento, alunos são capazes de aplicar conhecimentos conceituais existentes no problema para solucionar a atividade.

\section{Características da cognição no aprendizado de um ofício}

a) ela começa em uma tarefa enraizada em uma atividade familiar, legitima o conhecimento prévio dos alunos e serve de plataforma para eles adentrarem atividades não-familiares; b) mostra várias formas de solucionar um problema e que a heurística não é absoluta; c) torna os alunos ativamente envolvidos na cultura matemática ao permitir que eles construam suas próprias abordagens de soluções, reforçando o uso das ferramentas da cultura: 
vocabulário compartilhado, meios para discutir, refletir sobre, avaliar e validar os procedimentos da comunidade em um processo colaborativo.

A cognição do processo do aprendizado de ofício permite aos alunos o progresso de uma atividade enraizada para um princípio geral de uma cultura, em uma seqüência que começa com a modelagem em uma situação e o uso desta como plataforma para introduzir os alunos em uma atividade autêntica. Estas práticas levam a uma fase de controle mais autônomo e trabalho colaborativo, na qual eles constroem estratégias, linguagem e sistemas de crença. Neste processo de enculturação eles desenvolvem seu entendimento situado de um conhecimento conceitual em atividade.

De acordo com PALINCSAR, a idéia da aprendizagem cognitiva necessita "pesquisa prévia e acadêmica que seja relevante para as suas teses"; ela expressa uma preocupação que, para que a aprendizagem cognitiva (enquanto modelo da aprendizagem de ofício) seja adotada como um método de ensino na sala de aula, isto irá requerer um esforço adicional para esclarecer as diferenças entre cognição situada e didática e instrução descontextuada . O estudo atual da "aprendizagem cognitiva" ainda mostra necessidade que estudiosos advoguem uma educação direta (no contexto da prática do mundo real) e o cuidado por estratégias de aprendizagem.

PALINCSAR levanta uma importante questão, a de que ao "aprendizado cognitivo" falta uma seqüência clara e bem definida, ao longo de um estudo de seus objetivos e implicações no currículo, com a noção de currículo que temos hoje. No entanto, o trabalho de tornar essas idéias mais claras e aplicáveis mostra-se responsabilidade dos educadores, enquanto consigamos reconhecer a autenticidade dessas declarações e a possibilidade de uma mudança prática em nossa prática pedagógica.

\section{Aprendizagem e Cognição}

A abordagem da "aprendizagem cognitiva" percebe o conhecimento enquanto ferramenta, desenvolvendo conceitos através de atividade autêntica. Aprendizes de um ofício e aprendizes cognitivos aprendem de formas similares, adquirindo e desenvolvendo ferramentas através do trabalho e participação em seu campo de atividade. O papel da atividade é tão central ao aprendizado quanto o são a cultura e seu contexto. Na aprendizagem dos aprendizes de ofício, mestres modelam suas estratégias e tornam o seu conhecimento explícito dentro da atividade. Então eles endossam a autonomia do aprendiz para executar tarefas. Esta estrutura é similar ao paradigma da modelagem, orientação e afastamento gradual. (COLLINS; BROWN; NEWMAN, 1989). 
Interações sociais são muito importantes para circular o conhecimento, enquanto os aprendizes observam o praticantes em ação. Algumas características da natureza colaborativa do aprendizado são:

a) resolução de problemas coletiva, a qual gera, sinergicamente, soluções que não ocorreriam de outra forma;

b) mostra de papéis múltiplos - permite a observação de diferentes papéis, necessários para realizar a tarefa, enquanto a atividade está acontecendo;

c) confronta estratégias inefectivas e concepções errôneas;

d) possibilita habilidades de trabalho colaborativo, que são muito importantes para as condições do local de trabalho.

\section{Conclusão em direção a uma epistemologia de cognição situada}

Demandas por pesquisas que determinem o que deveria ser explicitado no ensino e o que deveria ser deixado implícito. A explicação do conhecimento pode deixar de fora representação indexadora, que é implícita e inerente ao contexto. O desafio será mostrar o que, na aprendizagem, é necessariamente implícito e deveria permanecer assim. "A prática educacional é vitima de uma epistemologia inadequada. Uma epistemologia que começa com atividade e percepção, que estão, antes de mais nada, enraizadas no mundo, pode simplesmente atropelar o problema clássico das representações conceituais mediadoras". Tanto WINERBURg quanto PALINCSAR esquecem-se de reconhecer o trabalho prévio sobre aprendizagem pelo fazer de John DEWEY (1927). Está claro, de fato, que os autores examinaram a natureza do conhe-cimento para entender a natureza da cognição do conhecimento. No entanto, este assunto oferece múltiplas perspectivas devido à ampla variedade de domínios de conhecimento e pede por um estudo extensivo em literatura relacionada para lhe fornecer um suporte mais amplo e acurado. A perspectiva histórica ajudará não apenas os autores a solucionarem as proposições problemáticas como aos leitores a situarem a história e desenvolvimento dessas idéias. Em nossa perspectiva, esse artigo mostra um problema estrutural, ele está mais bem definido e fundamentado em sua primeira parte, quando desenvolve conceitos para suportar suas declarações. Na segunda parte, no entanto, ele falha ao descrever e explicar o funcionamento da "aprendizagem cognitiva" como um método de ensino. Como modelo, ele se mostra ainda 
vago para ser implementado como uma abordagem pedagógica e requer um estudo mais específico se pretende substituir as práticas convencionais de ensino e escolarização.

\section{REFERÊNCIAS}

ALLEMAN, J.; BROPH, J. Activities as instructional tools: a framework for analysis and evaluation. Educational Researcher, p. 9-23, May 1991.

ANDERSON, J. R. The architecture of cognition in the architecture of cognition. Cambridge: Harvard University Press, 1983.

ANDERSON, R. C.; ARMBRUSTER, B. B. Some maxims for learning and instruction. Teachers College Record, v. 91, n. 3, p. 396-408, 1990.

BRANSFORD, J. D. et al. Anchored instruction: why we need it and how technololgy can help. In: NIX, D.; SPIRO R. (Ed.). (Volume unidentified). Hillsdale: Lawrence Erlbaum, [19-].

BROWN, J. S.; COLLINS, A.; DUGUID, P. Situated cognition and the culture of learning. Educational Researcher, v. 18, n. 1, p. 32-42, Jan./Feb. 1989.

BRUNER, J. Some elements of discovery. In: SHULMAN, L. S.; KEISLAR, E. R. (Ed.). Learning by discovery: a critical appraisal. Chicago: Rand McNally \& Co., 1966.

BURTON, R. R.; BROWN, J. S.; FISCHER, G. Skiing as a model of instruction. In: ROGOFF, B.; LAVE, J. (Ed.). Everyday cognition: Its development in social context. Cambridge: Harvard University Press.

COGNITION AND TECHNOLOGY GROUP AT VANDERBILT. Anchored instruction and its relationship to situated cognition. Educational Technology Research \& Development, v. 40, n. 1, p. 65-80, Aug./Sept. 1990.

COGNITION AND TECHNOLOGY GROUP AT VANDERBILT. The jasper experiment: an exploration of issues in learning and instructional design. Educational Technology Research \& Development, v. 40, n. 1, p. 65-80, 1991.

COLE, P.; WILSON, B. A review of cognitive teaching models. Educational Technology Research \& Development, v. 39, n. 4, p. 47-64, 1991.

COLLINS, A.; BROWN, J. S.; NEWMAN, S. E. Cognitive Apprenticeship: teaching the crafts of reading, writing, and mathematics. In: RESNICK, L. B. (Ed.). Knowing, learning and instruction: essays in honor of Robert Glaser. Hillsdale: Earlbaum, 1989. 
DILLNER, M. Using hypermedia to enhance content area instruction. Journal of Reading, v. 37, p. 260-270, 1993.

FARNHAM-DIGGORY, S. Learning processes. In: Cognitive processes in education. 2. ed. New York: Harper Collins Publishers, 1992. p. 83-118.

. The structure of cognition. In: Cognitive processes in education. New York: University of Delaware-Harper Collins, 1992.

FOSHAY, R. Sharpen up your schemata. Data Training, p. 18-25, May 1992.

JOHNSON-LAIRD, P. N. How is meaning mentally represented? In: ECO, U.; SATAMBROGIO, M.; VIOLI, P. (Ed.). Meaning and mental representations. 1988.

KOSMA, R. B. Learning with media. Review of Educational Research, v. 61, n. 2, p. 179-211, Summer 1991.

LAMPERT, M. Knowing, doing and teaching multiplication. Cognition and Instruction, n. 3, p. 305-342, 1986.

LAVE, J. Tailor-made experiments and evaluating the intellectual consequences of institute for Comparative Human Development, n. 1, p. 1-3, 1977.

. The culture of aquisition and the practice of understanding (IRL report 8800087). Palo Alto: Institute for Research on Learning, 1988.

. Cognition in practice. Boston: Cambridge, 1988.

. Word problems: a microcosm of theories of learning. Paper presented at AERA annual conference. New Orleans, 1988.

MILLER, G. A.; GILDEA, P. M. How children learn words. Scientific American, v. 3, n. 257, p. 94-99, 1987.

PALINCSAR, A. S. Metacognitive strategy instruction. Exceptional children, n. 53, p. 118-124, 1986.

; BROWN, A . L. Reciprocal teaching of comprehension fostering and monitoring activities. Cognition and Instruction, n. 1, p. 117-175, 1984.

RESNICK, L. B. Learning in school and out. Educational Researcher, p. 13-20, Dec. 1987.

SCARDAMALIA, M. B. C.; STEINBACH, R. Teachability of reflective processes in written composition. Cognitive Science, n. 8, p. 173-190, 1984.

SCHOENFELD, A. H. Mathematical problem solving. Orlando: Academic Press., 1985.

. On mathematics as sense-making: an informal attack on the unfortunate divorce of formal and informal mathematics. In: PERKINS, D. N.; SEGAL, J.; VOSS, J. (Ed.). Informal reasoning and education. Hillsdale: Erlbaum. 
SCHANK, R.; CHILDERS, P. Knowledge structures in the cognitive computer: on language, learning and artificial intelligence. Reading: Addison - Wesley, 1984. Chapter 6 .

SHAW, C. C. Problem-solving styles of students using software of the "second-and a-half-kind. Educational Technology, p. 14-20, July 1991.

SHUELL, T. J. Cognitive conceptions of learning. Review of Educational Research, v. 56, n. 4, p. 411-436, Winter 1986.

. Phases of meaningful learning. Review of Educational Research, v. 60, n. 4, p. 531-547, Winter 1990.

TAMASHINO, R.; BECHTELHEIMER, L. Expanding teacher's thinking in computer curriculum course. Educational Technology, p. 41-44, Feb. 1988.

WHITE, B. Y. Thinker tools: causal models, conceptual change, and science education. Boston: Technical Report, BBN Laboratories. 